\title{
Asthma and allergy patterns over 18 years after severe RSV bronchiolitis in the first year of life
}

\author{
Nele Sigurs, ${ }^{1}$ Fatma Aljassim, ${ }^{2,3}$ Bengt Kjellman, ${ }^{4}$ Paul D Robinson, ${ }^{5,6}$ \\ Fridrik Sigurbergsson, ${ }^{7}$ Ragnar Bjarnason, ${ }^{8}$ Per M Gustafsson ${ }^{2,4,9}$
}

\begin{abstract}
See Editorial, p 1033
- Additional tables are published online only. To view these files please visit the journal online (http://thorax.bmj. com).

${ }^{1}$ Department of Paediatrics, Borås Central Hospital, Borås, Sweden

${ }^{2}$ Queen Silvia Children's Hospital, Göteborg, Sweden ${ }^{3}$ Department of Paediatrics, Alwasl Hospital, Dubai Health Authority, Government of Dubai, UAE

${ }^{4}$ Department of Paediatrics, Central Hospital, Skövde,

Sweden

${ }^{5}$ Department of Respiratory Medicine, The Children's Hospital at Westmead, Australia ${ }^{6}$ The Children's Hospital at Westmead Clinical School, Discipline of Paediatrics and Child Health, Faculty of Medicine, University of Sydney, Australia

${ }^{7}$ Emergency Department, Landspitali University Hospital, Reykjavik, Iceland

${ }^{8}$ University of Iceland Department of Paediatrics, Landspitali University Hospital Iceland, Reykjavik, Iceland ${ }^{9}$ The Sahlgrenska Academy at the University of Gothenburg, Gothenburg, Sweden
\end{abstract}

\section{Correspondence to}

Dr Nele Sigurs, Department of Paediatrics, Borås Central Hospital, Borås S-50182, Sweden;

nele.sigurs@vgregion.se

Received 14 June 2009 Accepted 2 April 2010 Published Online First 27 June 2010

\section{ABSTRACT \\ Background An increased prevalence of asthma/} recurrent wheeze (RW), clinical allergy and allergic sensitisation up to age 13 years has previously been reported in subjects hospitalised with respiratory syncytial virus (RSV) bronchiolitis in their first year of life compared with matched controls. A study was undertaken to examine whether these features persist into early adulthood, to report longitudinal wheeze and allergy patterns, and to see how large and small airway function relates to RSV infection and asthma.

Methods Follow-up at age 18 years was performed in 46 of 47 subjects with RSV and 92 of 93 controls.

Assessments included questionnaire, clinical examination, skin prick tests, serum lgE antibodies to inhaled allergens, blood eosinophils, fraction of exhaled nitric oxide (FeNO), spirometry, multiple breath washout (lung clearance index, LCI) and dry air hyperventilation challenge.

Results Increased prevalence of asthma/RW (39\% vs $9 \%)$, clinical allergy (43\% vs $17 \%$ ) and sensitisation to perennial allergens (41\% vs 14\%) were present at age 18 in the RSV cohort compared with controls. Persistent/ relapsing wheeze associated with early allergic sensitisation predominated in the RSV cohort compared with controls (30\% vs 1\%). Spirometric function was reduced in subjects with RSV with or without current asthma, but not in asthmatic controls. LCl was linked only to current asthma, airway hyperresponsiveness and FeNO.

Conclusions Severe early RSV bronchiolitis is associated with an increased prevalence of allergic asthma persisting into early adulthood. Small airway dysfunction ( $\mathrm{LCI}$ ) is related to current asthma and airway inflammation but not to RSV bronchiolitis. Reduced spirometry after RSV may reflect airway remodelling.

\section{INTRODUCTION}

Respiratory syncytial virus (RSV) infection is the most common cause of severe bronchiolitis in infants. ${ }^{1}$ It is now established that early severe viral lower respiratory tract infections (LRTI) are frequently followed by recurrent wheeze, asthma and allergy during childhood. ${ }^{2} 3$

A number of previous studies have investigated the association between RSV infection in infancy and later development of asthma, although differences in timing and severity of RSV infection exist between studies. The Tucson Children's Respiratory Study (a birth cohort study) reported an increased prevalence of respiratory symptoms until age 11 and residual impaired lung function at age 13 in those experiencing a relatively mild RSV LRTI (not requiring hospitalisation) during the first 3 years of life. ${ }^{4}$ Impaired lung function at age 10 has also been reported following hospitalisation in the first year with proven LTRI. ${ }^{5}$ Recently, data from a cohort previously hospitalised in the first 2 years of life and followed up at 18-20 years suggested that this impairment may persist into early adulthood. ${ }^{6}$ Histopathology studies of fatal RSV bronchiolitis demonstrate extensive damage to the airway epithelium and marked small airway obstruction, ${ }^{7}$ yet the presence of persistent small airway damage in later life is unclear. In adult asthma, small airway dysfunction is closely associated with bronchial hyper-responsiveness, ${ }^{8} 9$ a fundamental component of asthma correlated to disease severity. ${ }^{10}$

Our cohort represents infants aged $<1$ year with severe primary RSV bronchiolitis (43/47 $\leq 6$ months of age at hospitalisation). We have previously demonstrated increased prevalence of asthma or recurrent wheeze (RW) and allergic sensitisation compared with a matched control cohort at ages 3 , 7 and 13 years. ${ }^{11-13}$ In this paper we present the 18-year follow-up data together with a longitudinal analysis of wheeze and allergy patterns. The primary aim was to see if the higher prevalence of asthma or RW, clinical allergy and allergic sensitisation persist at age 18. The secondary aims were to describe the wheeze patterns in both cohorts and to investigate how large and small airway function relate to severe RSV bronchiolitis, current asthma, airway hyper-responsiveness (AHR) and markers of allergic inflammation.

\section{METHODS}

The study design is summarised in table 1. Fortyseven children aged $<1$ year hospitalised with RSV LRTI $^{14}$ between December 1989 and April 1990 constituted the index group. An age- and gendermatched control group ( $\mathrm{n}=93$ ) was recruited from children attending the same child healthcare centres as the index cases. Both cohorts were followed up prospectively at ages 1, 3, 7, 13 and 18 years. Diagnosis of bronchiolitis was originally based on criteria published by Court, ${ }^{15}$ but was also consistent with other later published criteria. ${ }^{16}$ Demographic details at age 18 are given in table 2

Structured questionnaires were completed at all follow-up time points to record demographic, hereditary and clinical symptoms of allergy or wheeze. A clinical examination was also performed The investigations performed at each assessment are summarised in table 1. Details on skin prick tests (SPT) and serum IgE tests are given in our 
Table 1 Study design and proportion of the 47 children in the RSV cohort and the 93 in the control cohort seen at follow-up during the study period

\begin{tabular}{|c|c|c|c|c|c|c|}
\hline & \multicolumn{6}{|l|}{ Age (years) } \\
\hline & 1 & $1.5,2$ and 2.5 & 3 & 7 & 13 & 18 \\
\hline Total number (RSV, controls) & 47,93 & 47,93 & 47,93 & 47,93 & 46,92 & 46,92 \\
\hline$\%$ of cohort (RSV and controls) & 100 & 100 & 100 & 100 & 98.5 & 98.5 \\
\hline Examination & $\times$ & & $\times$ & $\times(46,89)$ & $\times(45,89)$ & $\times(42,87)$ \\
\hline Skin prick tests (RSV, controls) & $\times(47,92)$ & & $\times(45,92)$ & $\times(44,89)$ & $\times(42,87)$ & $\times(41,85)$ \\
\hline Egg white & $\times$ & & $\times$ & $\times$ & & \\
\hline Horse & & & & $x$ & $\times$ & $x$ \\
\hline Birch & & & $x$ & $x$ & $\times$ & $x$ \\
\hline Timothy & & & & $x$ & $x$ & $x$ \\
\hline Mugworth & & & & & $x$ & $x$ \\
\hline HDM (D pteronyssinus) & & & $x$ & $\times$ & $\times$ & $x$ \\
\hline $\lg E$ to food allergens (Fx5) & & & $\times$ & $\times$ & & \\
\hline IgE to inhaled allergens (Phadiatop) & & & $x$ & $x$ & $x$ & $\times(41,85)$ \\
\hline Blood eosinophil counts & & & & & & $\times(40,86)$ \\
\hline Lung function tests & & & & $\times(44,86)$ & $\times(44,86)$ & $\times(42,86)$ \\
\hline Spirometry & & & & $\times$ & $\times$ & $\times$ \\
\hline Multiple breath washout (LCI) & & & & & & $\times(41,86)$ \\
\hline FeNO & & & & & & $\times(42,86)$ \\
\hline DACh & & & & & $\times(43,86)$ & $\times(41,86)$ \\
\hline
\end{tabular}

Numbers in parentheses (RSV, controls) if differed from numbers followed up at that time point.

A skin prick test weal with a mean diameter $\geq 3 \mathrm{~mm}$ was regarded positive. Phadiatop (Pharmacia Upjohn Diagnostics AB, Uppsala, Sweden) is a screening test for antibodies to inhaled allergens. Fx5 (Pharmacia Upjohn Diagnostics AB, Uppsala, Sweden) is a screening test for food allergens.

$\mathrm{HDM}$, house dust mite; DACh, dry air hyperventilation challenge; LCl, lung clearance index; FeNO, fractional exhaled nitric oxide; RSV, respiratory syncytial virus.

previous publications. ${ }^{11-13}$ The specific IgE assay method used (ImmunoCAP) was consistent throughout the study period.

Spirometry was performed at baseline from age 7 and postbronchodilator at ages 13 and 18 years, according to American Thoracic Society recommendations, ${ }^{17}$ and expressed as standard deviation scores (z-scores) using recently published normative data. ${ }^{18} 19$ Inhaled long-acting $\beta_{2}$ agonists were withheld for $24 \mathrm{~h}$ and short-acting $\beta_{2}$ agonists or cromoglycates for $6 \mathrm{~h}$ prior to testing. Inhaled corticosteroids (ICS), if used, were not discontinued. Isocapnic dry air hyperventilation challenge (DACh) was performed after baseline spirometry at age 13 and 18 years (described in more detail in the online supplement). At age 18, prior to these lung function tests, the fraction of nitric oxide in expired air (FeNO) was measured in duplicate at an expiratory flow of $50 \mathrm{ml} / \mathrm{s}$ using the Niox Mino (Aerocrine, Stockholm, Sweden) and the mean FeNO result reported.

Multiple-breath washout (MBW) was performed before spirometry using sulfur hexafluoride $\left(\mathrm{SF}_{6}\right)$ as the marker gas and

Table 2 Demographic data and family history of the respiratory syncytial virus (RSV) and control groups at age 18

\begin{tabular}{lll}
\hline Variable & RSV (n=46) & Controls $(\mathbf{n}=\mathbf{9 2})$ \\
\hline Weight $(\mathrm{kg})$ & $65(12)$ & $70(13)$ \\
Height $(\mathrm{cm})$ & $174(10)$ & $174(10)$ \\
Parental atopy & $30 / 46(65 \%)$ & $52 / 92(56 \%)$ \\
Parental asthma & $18 / 46(39 \%)$ & $25 / 92(27 \%)$ \\
Tobacco smoke exposure & & \\
$\quad$ Active & $10 / 45(22 \%)$ & $14 / 92(15 \%)$ \\
$\quad$ Passive (family member) & $19 / 45(42 \%)$ & $40 / 92(43 \%)$ \\
Indoor furred pets & $24 / 46(52 \%)$ & $56 / 92(61 \%)$ \\
\hline \multicolumn{2}{l}{$\begin{array}{l}\text { Mean (SD) or proportions (percentages) of subjects are given. No significant differences } \\
\text { were seen between the groups. }\end{array}$}
\end{tabular}

Late onset

Persistent/relapsing

a mass spectrometer for gas analysis and as previously described in detail elsewhere. ${ }^{20}$ Lung clearance index (LCI) was calculated as the number of lung volume turnovers (ie, the cumulative expired volume divided by the functional residual capacity) needed to lower the end-tidal tracer gas concentration to $1 / 40$ th of the starting concentration. A high value of LCI thus indicates abnormal ventilation distribution. The mean LCI result from three MBWs in each subject was reported. In a previous study including healthy subjects, the mean, SD and upper limit of

Table 3 Wheeze patterns defined from the presence/absence $(+/-)$ of asthma/recurrent wheeze at four follow-up stations and the numbers of cases in each wheeze pattern group for each cohort

\begin{tabular}{lllllll}
\hline & \multicolumn{7}{l}{ Age (years) } & & \\
\cline { 2 - 5 } Wheeze pattern & $\mathbf{3}$ & $\mathbf{7}$ & $\mathbf{1 3}$ & $\mathbf{1 8}$ & $\mathbf{R S V}(\mathbf{n}=\mathbf{4 6})$ & Controls (n=92) \\
\hline Never wheeze & - & - & - & - & $14(3,11)^{* * *}$ & $69(27,42)$ \\
Transient wheeze & + & - & - & - & $7(5,2)$ & $11(8,3)$ \\
Remitting/intermittent & - & - & + & - & $7(4,3)$ & $4(2,2)$ \\
& - & + & - & - & & \\
& - & + & + & - & & \\
Late onset & + & + & - & - & & \\
Persistent/relapsing & + & + & + & - & & \\
& - & - & + & + & $4(1,3)$ & $7(3,4)$ \\
& - & - & - & + & & \\
& + & + & + & + & $14(7,7)^{* * *}$ & $1(1,0)$ \\
& - & + & + & + & & \\
& + & + & - & + & & \\
\hline
\end{tabular}

Male, female distribution shown in parentheses.

Statistical evaluation refers to comparison of proportion of subjects in the different wheeze pattern groups in the RSV and control cohorts: ${ }^{* *} p<0.001$.

$\mathrm{RSV}$, respiratory syncytial virus. 
normality (ULN; mean + 1.96 SD) for LCI were 6.33, 0.43 and 7.17, respectively. ${ }^{20}$

At each follow-up time point, asthma was defined as $\geq 3$ episodes of physician-verified wheeze and RW as $\geq 3$ episodes of parent-reported wheeze. Definitions of the wheezing patterns over time are shown in table 3. Allergic rhinoconjunctivitis (ARC) or clinical allergy was defined as rhinitis/conjunctivitis occurring at least twice following exposure to a particular allergen and unrelated to infection. Atopic dermatitis (AD) was defined as a pruritic, chronic or chronically relapsing non-infectious dermatitis. Allergic sensitisation implied occurrence of $\operatorname{IgE}$ antibodies estimated by SPT and/or serum IgE tests. Current disorder denotes symptoms over the last 12 months. Active and passive smoke exposures, the presence of pets in the household, and atopic or allergic heredity were assessed by questionnaire. A positive history of atopic disease (AD, ARC or asthma) in firstdegree relatives (parents or siblings) was based on physician diagnosis.

\section{Statistical analyses}

Yates' corrected $\chi^{2}$ test was used for estimation of differences in prevalence among groups and subgroups. One-way ANOVA was used for parametric continuous variables and Tukey HSD for subsequent group comparisons, if the overall F-test was significant. A Mann-Whitney test was used to test group differences for non-parametric continuous variables. 95\% CIs for mean and median differences were calculated. $\chi^{2}$ tests for trend were used to assess the combined influence of group allocation (RSV vs control) and a parental history of physician-diagnosed asthma on asthma/RW, ARC and sensitisation at age 18. Pearson correlation tests were used to assess correlation of parametric data, while the Spearman rank test was used for non-parametric data. Kaplan-Meier survival analysis was used to compare time free from diagnosis of asthma, ARC, positive Phadiatop test or positive SPT to any perennial allergen at a follow-up station; $p$ values based on Mantel-Cox log ranks were calculated. Multivariate logistic regression analyses were performed to establish risk factors for asthma/RW, asthma alone and ARC at age 18. ORs with $95 \% \mathrm{CI}$ and $\mathrm{p}$ values were reported. Additionally, conditional logistic regression tests were performed to adjust for any degree of residual confounding due to the constitution of the groups during the study period. SPSS Version 15.0 software for Windows (SPSS Inc, Chicago, Illinois, USA), SPSS SamplePower 2.0 software and CI Analysis software version 2.1.2 (Trevor Bryant, University of Southampton) were used for the statistical analyses.

\section{RESULTS}

Forty-six of the 47 subjects with RSV and 92 of the 93 controls were followed up to 18 years of age. The demographic details of the two cohorts at age 18 are summarised in table 2, and from age 1-18 years in table E1 in the online supplement. No significant differences were seen at age 18 .

\section{Cross-sectional data at age $\mathbf{1 8}$}

Current asthma/RW was documented in 18 of 46 (39\%) subjects with RSV and 8 of $92(9 \%)$ controls $(p=0.001)$. Current asthma alone was found in 15 of 46 (33\%) subjects with RSV and 6 of 92 $(7 \%)$ controls $(p<0.001)$. ARC was diagnosed in 20 of 46 subjects with RSV (43\%) and 16 of $92(17 \%)$ controls $(p=0.002)$. No difference was found in the prevalence of $\mathrm{AD}$ (5 of $46(11 \%)$ subjects with RSV vs 8 of 92 (9\%) controls).

The prevalence of sensitisation determined by SPT was significantly increased in the RSV cohort compared with controls to any animal dander (cat, dog, horse) (33\% vs $11 \%$; $\mathrm{p}=0.005$ ) or any perennial (animal danders and house dust mites $(\mathrm{HDM})$ ) (41\% vs $14 \% ; \mathrm{p}=0.001)$ (see table $\mathrm{E} 2$ in online supplement). A higher prevalence of positive Phadiatop responses was seen in the RSV cohort ( $56 \%$ vs $28 \%$, $\mathrm{p}=0.005$ ) (see table E2 in online supplement). For the whole cohort, the most commonly identified specific IgE antibodies performed in those testing positive to Phadiatop screening were to 'any perennial' (39 of 126, 31\%), and were significantly increased in subjects with RSV compared with controls ( $51 \%$ vs $21 \%$; $\mathrm{p}=0.001$ ).

Airway function data are summarised in table 4. Reduced spirometric airway function (forced expiratory volume in $1 \mathrm{~s}$ $\left(\mathrm{FEV}_{1}\right)$, ratio of $\mathrm{FEV}_{1}$ to forced vital capacity (FVC) and forced expiratory flow at $25-75 \% \mathrm{FVC}\left(\mathrm{FEF}_{25-75}\right)$ ) was documented in the RSV cohort compared with controls, but LCI did not differ. AHR, bronchodilator response and blood eosinophil cell counts were greater in the RSV cohort than in the controls, but FeNO

Table 4 Airway function and inflammatory markers in RSV and control groups at age 18 years

\begin{tabular}{|c|c|c|c|}
\hline & $\operatorname{RSV}(n=46)$ & Control $(n=92)$ & 95\% $\mathrm{Cl}$ for difference \\
\hline \multicolumn{4}{|l|}{ Resting } \\
\hline $\mathrm{FEV}_{1}$ (z-score) & $-0.28(0.93)$ & $0.11(1.08)$ & 0.00 to $0.7^{*}$ \\
\hline $\mathrm{FEV}_{1} / \mathrm{FVC}$ (z-score) & $-0.68(0.85)$ & $-0.08(1.03)$ & 0.24 to $0.97^{* *}$ \\
\hline $\mathrm{LCl}$ & $6.63(0.52)$ & $6.59(0.43)$ & -0.21 to 0.13 \\
\hline \multicolumn{4}{|l|}{ Challenge (DACh) } \\
\hline Fall in $\mathrm{FEV}_{1}(\%)$ & $4.7(0.0-37.8)$ & $3.1(0.0-42.8)$ & 0.0 to $3.2^{*}$ \\
\hline $\mathrm{FEV}_{1}$ (z-score) & $0.16(0.71)$ & $0.38(1.30)$ & -0.18 to 0.61 \\
\hline $\mathrm{FEV}_{1} / \mathrm{FVC}$ (z-score) & $-0.04(0.88)$ & $0.42(0.95)$ & 0.11 to $0.80^{* *}$ \\
\hline $\mathrm{FEF}_{25-75}$ (z-score) & $-0.01(0.78)$ & $0.38(0.83)$ & 0.09 to $0.69^{*}$ \\
\hline \multicolumn{4}{|l|}{ Inflammatory markers } \\
\hline FeNO (ppb) & $14(6-84)$ & $13(5-74)$ & -1 to 5 \\
\hline
\end{tabular}

Data are shown as mean (SD) or median (range).

For normally distributed data, differences between the two groups are shown with $95 \% \mathrm{Cl}$ for the mean values and for non-normally distributed data as the $95 \% \mathrm{Cl}$ for the medians. ${ }^{*} \mathrm{p}<0.05,{ }^{* *} \mathrm{p}<0.01,{ }^{* * *} \mathrm{p}<0.001$.

For exact number of subjects in RSV and control group performing each test, see Table 1.

DACh, isocapnic dry air hyperventilation challenge; $\mathrm{FEF}_{25-75}$, forced expiratory fraction at $25-75 \%$ forced vital capacity; FeN0, fraction exhaled nitric oxide; FEV $\mathrm{B}_{1}$, forced expiratory volume in $1 \mathrm{~s}$; FVC, forced vital capacity; LCl, lung clearance index; ppb, parts per billion; RSV, respiratory syncytial virus. 
Table 5 Airway function and inflammatory markers in RSV versus control subjects with or without current asthma/RW at age 18 years

\begin{tabular}{|c|c|c|c|c|c|c|}
\hline & \multicolumn{3}{|c|}{ Current asthma/RW } & \multicolumn{3}{|c|}{ No current asthma/RW } \\
\hline & $\operatorname{RSV}(n=18)$ & Controls $(n=8)$ & 95\% CI for difference & RSV $(n=24)$ & Controls $(n=78)$ & 95\% $\mathrm{Cl}$ for difference \\
\hline \multicolumn{7}{|l|}{ Resting } \\
\hline $\mathrm{FEV}_{1}$ (z-score) & $-0.64(0.85)$ & $0.05(0.77)$ & -0.03 to 1.42 & $0.00(0.91)$ & $0.11(1.11)$ & 0.00 to $0.77^{*}$ \\
\hline $\mathrm{FEF}_{25-75}$ (z-score) & $-0.89(0.71)$ & $-0.06(0.86)$ & 0.17 to $1.50^{*}$ & $-0.38(0.75)$ & $-0.05(0.85)$ & 0.25 to $0.86^{* * *}$ \\
\hline LCl & $6.88(0.63)$ & $6.89(0.25)$ & -0.46 to 0.49 & $6.44(0.31)$ & $6.56(0.43)$ & -0.21 to 1.29 \\
\hline \multicolumn{7}{|l|}{ Challenge (DACh) } \\
\hline \multicolumn{7}{|l|}{ Post-bronchodilation } \\
\hline Rise in $\mathrm{FEV}_{1}(\%)$ & $6.5(0.0-10.2)$ & $2.5(0.0-9.9)$ & -0.3 to 6.3 & $5.4(0.0-10.5)$ & $3.1(0.0-10.4)$ & -0.0 to $3.2^{* *}$ \\
\hline $\mathrm{FEV}_{1}$ (z-score) & $-0.18(0.79)$ & $0.25(1.05)$ & -0.34 to 1.20 & $0.41(0.81)$ & $0.39(1.15)$ & -0.18 to 0.61 \\
\hline $\mathrm{FEV}_{1} / \mathrm{FVC}$ (z-score) & $-0.23(0.83)$ & $0.48(1.06)$ & -0.09 to 1.50 & $0.11(0.90)$ & $0.41(0.95)$ & 0.11 to $0.80^{* *}$ \\
\hline $\mathrm{FEF}_{25-75}$ (z-score) & $-0.29(0.76)$ & $0.48(0.72)$ & 0.11 to $1.43^{*}$ & $0.19(0.74)$ & $0.37(0.84)$ & 0.11 to $0.69 *$ \\
\hline \multicolumn{7}{|l|}{ Inflammatory markers } \\
\hline
\end{tabular}

Data are shown as mean (SD) or median (range).

For normally distributed data differences between the two groups are shown with $95 \% \mathrm{Cl}$ for the mean values and for non-normally distributed data as the $95 \% \mathrm{Cl}$ for the medians ${ }^{*} \mathrm{p}<0.05$, ${ }^{* *} \mathrm{p}<0.01$, ${ }^{* * *} \mathrm{p}<0.001$.

DACh, isocapnic dry air hyperventilation challenge; $\mathrm{FEF}_{25-75}$, forced expiratory fraction at $25-75 \%$ forced vital capacity; FeNO, fraction exhaled nitric oxide; FEV $\mathrm{F}_{1}$, forced expiratory volume in $1 \mathrm{~s}$; FVC, forced vital capacity; LCI, lung clearance index; ppb, parts per billion; RSV, respiratory syncytial virus; RW, recurrent wheeze.

was not. $\mathrm{FEV}_{1} / \mathrm{FVC}$ and $\mathrm{FEF}_{25-75}$ remained lower in the RSV cohort after bronchodilation. Subjects with RSV had lower spirometry results than the controls, irrespective of current asthma/RW diagnosis (table 5). Spirometry findings were similar in controls with or without current asthma/RW. LCI was significantly raised in subjects with RSV $(\mathrm{p}=0.006)$ and controls $(p=0.033)$ with current asthma/RW compared with corre- sponding subjects without asthma (see table E3 in online supplement). Only LCI differed significantly between the controls with and those without current asthma/RW (see table E4 in online supplement). In the RSV cohort, the maximum percentage fall in $\mathrm{FEV}_{1}$ after dry air challenge correlated significantly with LCI $\left(\mathrm{r}^{2}=0.44 ; \mathrm{p}<0.001\right)$, FeNO levels $\left(\mathrm{r}^{2}=0.26\right.$; $\mathrm{p}<0.001)$ and with blood eosinophil counts $\left(\mathrm{r}^{2}=0.12 ; \mathrm{p}=0.011\right)$,
A

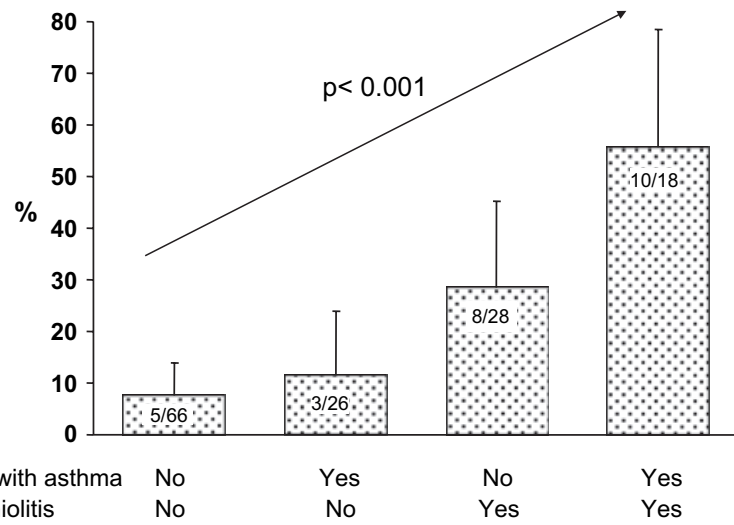

C

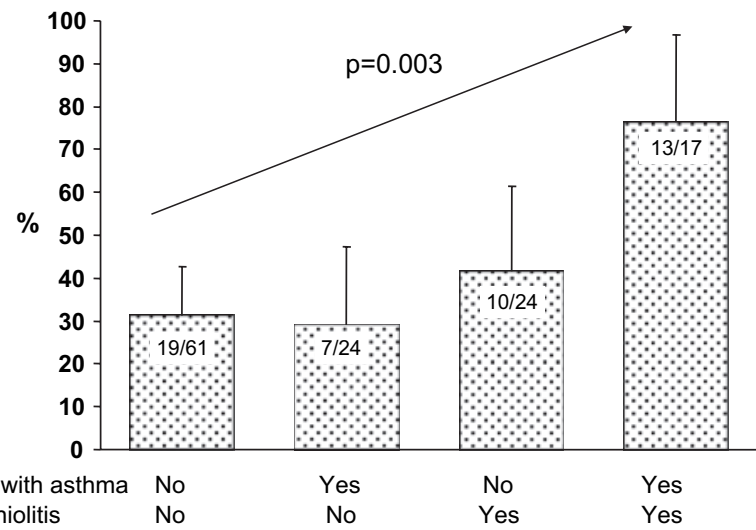

B

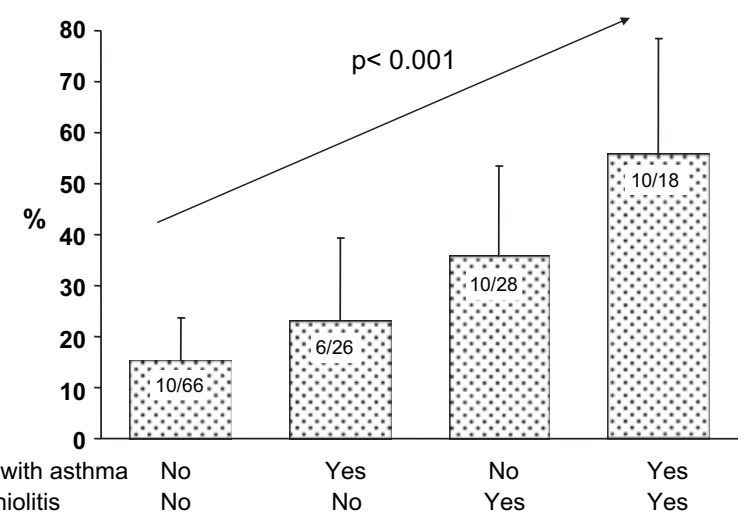

D

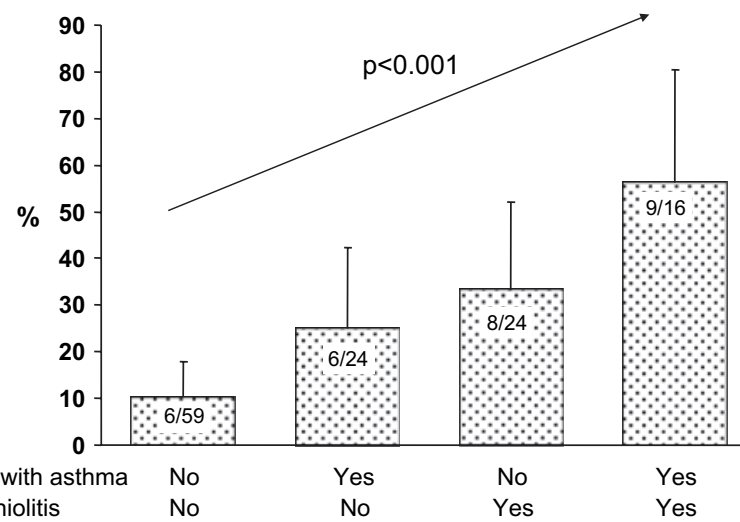

Figure 1 Proportion (\%) with (A) current asthma/recurrent wheeze, (B) current allergic rhinoconjunctivitis, (C) current positive Phadiatop test and (D) current positive skin prick test to perennial allergens in respiratory syncytial virus (RSV) and control cohorts with respect to heredity for asthma. $\chi^{2}$ test for trend was used in all graphs and error bars denote $95 \% \mathrm{Cl}$. 
but not with spirometry results (see table E5 in online supplement). Among the subjects with current asthma/RW, regression analyses also demonstrated significant relationships between LCI and the maximum percentage fall in $\mathrm{FEV}_{1}$ after dry air challenge $\left(r^{2}=0.26 ; p=0.005\right)$ and between LCI and FeNO $\left(\mathrm{r}^{2}=0.29 ; \mathrm{p}=0.003\right)$.

\section{Family history and other risk factors for asthma and ARC at age 18}

A family history of asthma and atopy did not differ between the RSV and control cohorts at age 18. A number of risk factors for current asthma/RW, current asthma and current ARC, respectively, were assessed using multivariate logistic regression analyses including both cohorts. For current asthma/RW and for current asthma, the risk factors included were: allocation (RSV/ control), gender, domestic furred pets in the first year of life, parental smoking in the first year of life, own smoking at age 18, current ARC of the subjects themselves, physician-diagnosed parental ARC and physician-diagnosed parental asthma.

For current asthma/RW, only RSV (OR 6.2; 95\% CI 2.0 to $19.2 ; \mathrm{p}<0.001)$ and current ARC of the subjects (OR 6.1; 95\% CI 2.1 to $18.1 ; p<0.001)$ were significant independent risk factors. For current asthma alone, similar results were found (RSV: OR 7.2 (95\% CI 2.1 to 23.9; p<0.001); current ARC: OR 4.4 (95\% CI 1.4 to $14.0 ; \mathrm{p}<0.001)$ ).
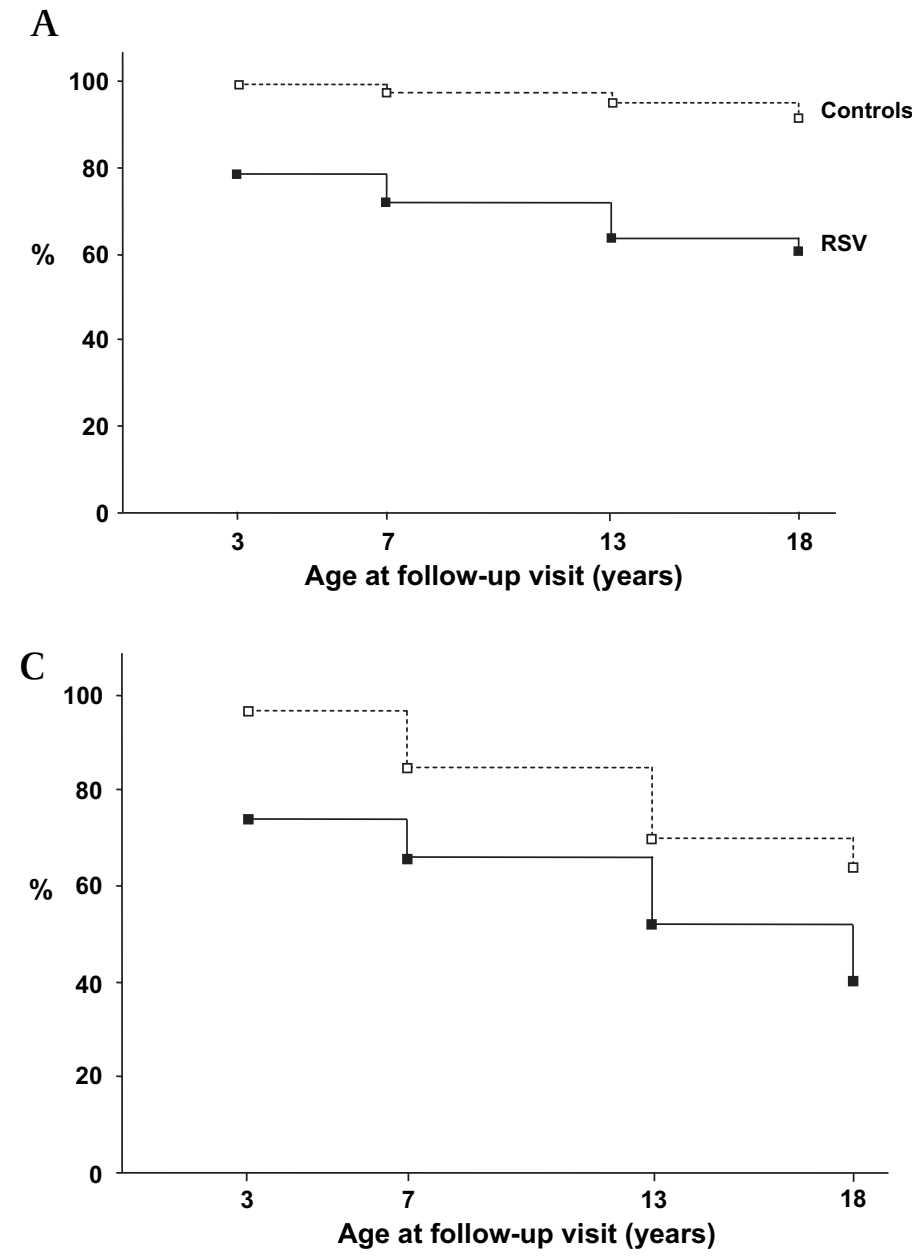

The risk factors included in the analysis for current ARC were the same, except for current ARC of the subjects. Only RSV (OR 3.6; 95\% CI 1.6 to 8.5; $\mathrm{p}=0.003$ ) was a significant independent risk factor. Corresponding conditional logistic regression tests gave similar results (see table $\mathrm{E} 6$ in the online supplement).

Numerically, the RSV subgroup with a parental history of asthma had a higher prevalence of asthma/RW, ARC and positive sensitisation than the RSV subgroup without parental asthma, but no significant differences were found $(p=0.058-0.308)$. The statistical power to detect significant differences between the RSV subgroups was $<50 \%$. For the two cohorts analysed together, however, the combination of RSV allocation and history of parental asthma resulted in significant trends for disease and sensitisation (figure $1 \mathrm{~A}-\mathrm{D}$ ).

\section{Longitudinal data over the entire study period: subjects with RSV versus controls}

Kaplan-Meier survival plots for time free from asthma diagnosis, ARC diagnosis, positive Phadiatop test or positive SPT are shown in figure $2 \mathrm{~A}-\mathrm{D}$. The RSV group had significantly shorter time free from these diagnoses and test findings.

Reduced lung function in the RSV cohort was evident from the age of 7 years (mean (SD) baseline $\mathrm{FEF}_{25-75}$ z-scores in RSV
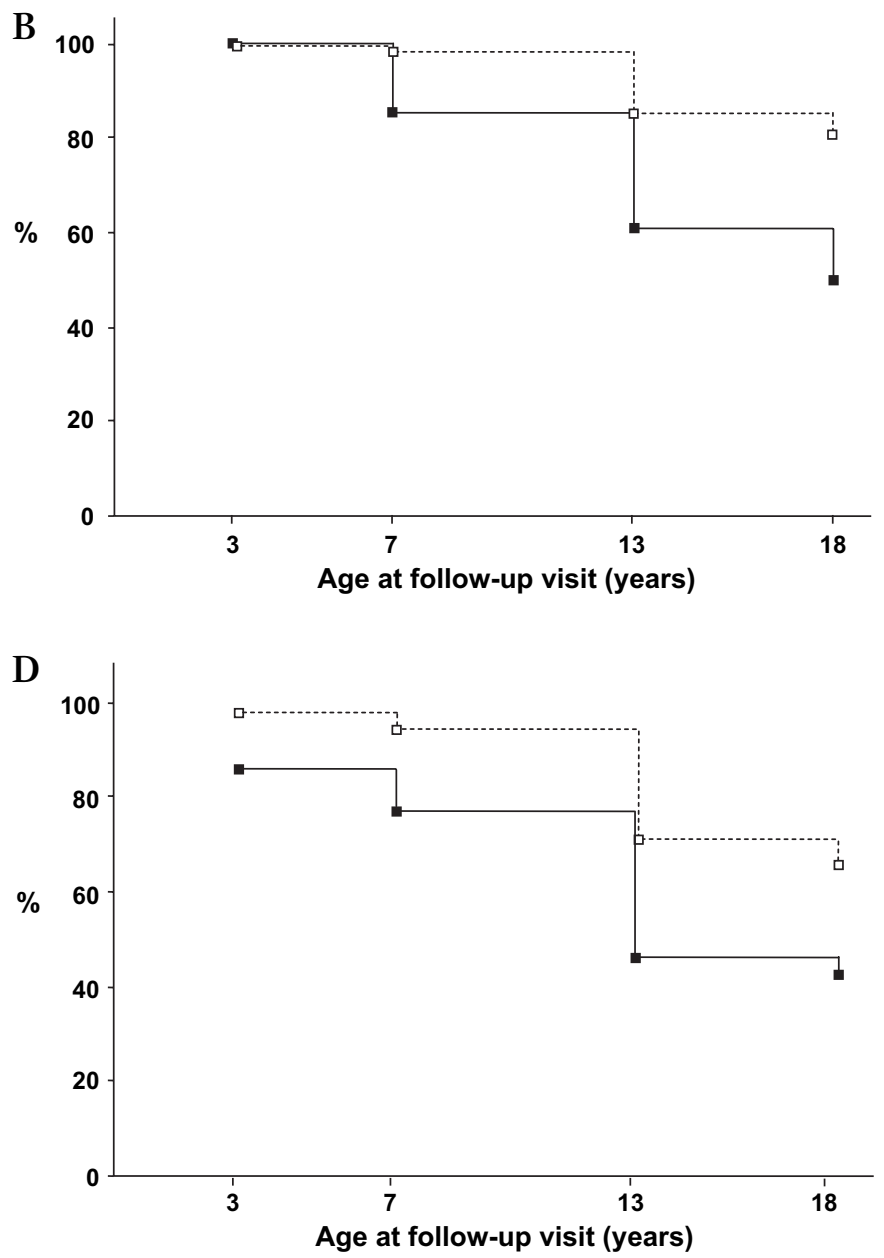

Figure 2 Proportion (\%) of subjects in the respiratory syncytial virus and control cohorts who never had (A) an asthma diagnosis at follow-up at ages $3,7,13$ and 18 years (log rank (Mantel-Cox) $\left.\chi^{2} 21.0 ; \mathrm{df} 1 ; \mathrm{p}<0.001\right) ;(\mathrm{B})$ an allergic rhinoconjunctivitis diagnosis at follow-up at ages $3,7,13$ and 18 years (log rank (Mantel-Cox) $\left.\chi^{2} 15.5 ; \mathrm{df} 1 ; \mathrm{p}<0.001\right)$; (C) a positive Phadiatop test at follow-up at ages 3, 7, 13 and 18 years (log rank (Mantel-Cox) $\left.\chi^{2} 8.3 ; \mathrm{df} 1 ; p=0.004\right)$; and (D) a positive skin prick test at follow-up at ages $3,7,13$ and 18 years (log rank (Mantel-Cox) $\chi^{2} 8.0 ;$ df $\left.1 ; p=0.005\right)$. 
group vs controls at age $7:-0.63(1.04)$ vs $-0.22(0.95)(95 \% \mathrm{CI}$ 0.05 to $0.77 ; \mathrm{p}=0.025)$; at age 13 : $-0.58(0.77)$ vs $-0.12(0.78)$ (95\% CI 0.17 to $0.74 ; p=0.002)$. At ages 13 and 18 a greater fall in $\mathrm{FEV}_{1}$ after DACh was seen in subjects with RSV. ${ }^{13}$

\section{Wheeze patterns up to age 18 years}

The persistent/relapsing asthma/RW pattern occurred more frequently in the RSV cohort with fewer non-wheezers in the RSV group (table 3). No difference in other wheeze patterns was seen. All 14 individuals with a persistent/relapsing asthma/RW pattern in the RSV cohort had current symptoms on at least two time points (seven on all four and six on three occasions). Asthma/RW was noted from age 3 in 11 of 14 subjects in this group. The one individual in the control group with persistent/ relapsing asthma had symptoms at all occasions from age 7. Nine subjects with RSV (7 with persistent/relapsing asthma/ RW and 2 with late onset asthma/RW) and 3 control subjects with late-onset asthma/RW were treated with ICS ( $\mathrm{p}=0.002)$. The persistent/relapsing group (14 RSV and 1 control) was characterised by the highest prevalence of co-existing ARC (figure $3 \mathrm{~A}$ ) and sensitisation (figure $3 \mathrm{~B}$ ). The significantly higher prevalence of sensitisation was evident at ages 3, 7 and 13, and similarly for ARC at age 7 and 13 (data not shown).

At 18 years of age the persistent/relapsing wheeze group had significantly lower airway function $\left(\mathrm{FEV}_{1}, \mathrm{FEV}_{1} / \mathrm{FVC}\right.$ and $\mathrm{FEF}_{25-75} \mathrm{z}$-scores), higher LCI and AHR than the non-wheeze group (table 6). Bronchodilator response, FeNO and blood eosinophil counts were significantly increased compared with never wheezers only in the persistent/relapsing asthma/RW group.

\section{DISCUSSION}

The previously reported over-representation of asthma (and asthma/RW), clinical allergy and allergic sensitisation in this RSV cohort persisted into early adulthood. The high prevalence of current asthma/RW in the RSV cohort was due to a high proportion of subjects with early-onset allergy-associated wheezing persisting through childhood and adolescence, and was accompanied by reduced airway function, elevated FeNO and eosinophil counts. A history of hospitalisation for RSV bronchiolitis was the only significant risk factor identified at age 18 for current asthma/RW, asthma alone or ARC.

At age 18, spirometry results were reduced in subjects with RSV with or without current asthma/RW compared with corresponding controls, but subjects with RSV without current asthma did not show evidence of small airway dysfunction as measured by LCI. Spirometry results showed no relationship with AHR or markers of allergic inflammation. In contrast, small airway dysfunction (LCI) correlated with current asthma, AHR and FeNO, a marker of ongoing allergic airway inflammation.

This controlled follow-up study and its findings are unique. It describes the development of asthma and allergy prospectively after severe primary RSV bronchiolitis in the first year life, and has involved several follow-up time points from infancy to young adulthood, all with very high attendance rates. The subsequent high frequency of early-onset allergic asthma persisting to age 18 has not previously been reported. Only two other prospective studies of RSV have documented a high prevalence of early sensitisation comparable to ours at 1 year ${ }^{21}$ and 3 years of age. ${ }^{22}$

The debate between a causal or genetic predisposition relationship between RSV and asthma has continued for decades and cannot be answered by this study. Two recent large registrybased studies have attempted to answer this question, with each declaring opposite conclusions. ${ }^{23} 24$ The weaknesses in the study designs and the difficulties in answering this question have been highlighted in a recent editorial. ${ }^{25}$ RSV-positive infants with wheezing may include both those with primary RSV LRTI or those with an existing non-atopic or atopic wheezing disorder exacerbated by RSV. In our cohort, $91 \%$ of the index subjects were 6 months or younger when admitted and only one had a previous history of lower airway symptoms, which strongly suggests that our cohort comprises subjects with early primary severe RSV LRTI. It is thought that early infancy, with a relatively Th-2 skewed immune system, may constitute a particularly vulnerable period of life for subsequent development of asthma following severe viral LRTI. ${ }^{3}$ Viral airway infections and atopy may interact in a multiplicative way to promote asthma development in young childhood. ${ }^{26}$ In addition, predisposition to both early severe RSV bronchiolitis and allergic sensitisation may be related to the interleukin (IL)-13/IL-4 gene locus. ${ }^{27}$ Potential hereditary susceptibility to RSV bronchiolitis has also been reported. ${ }^{28}$

Potential confounding factors do exist within our cohort. Mallia and Johnston ${ }^{29}$ have previously suggested that the higher rate of asthma in our RSV cohort compared with controls could be due to selection bias generated by the selection of controls contemporaneously from the same child healthcare centres as the index cases. Theoretically, our selection process during an ongoing RSV epidemic could generate controls with a lower susceptibility for severe RSV bronchiolitis and a lower risk for subsequent allergic asthma if severe RSV bronchiolitis and subsequent allergic asthma are markers of the same genotype. There are two main reasons

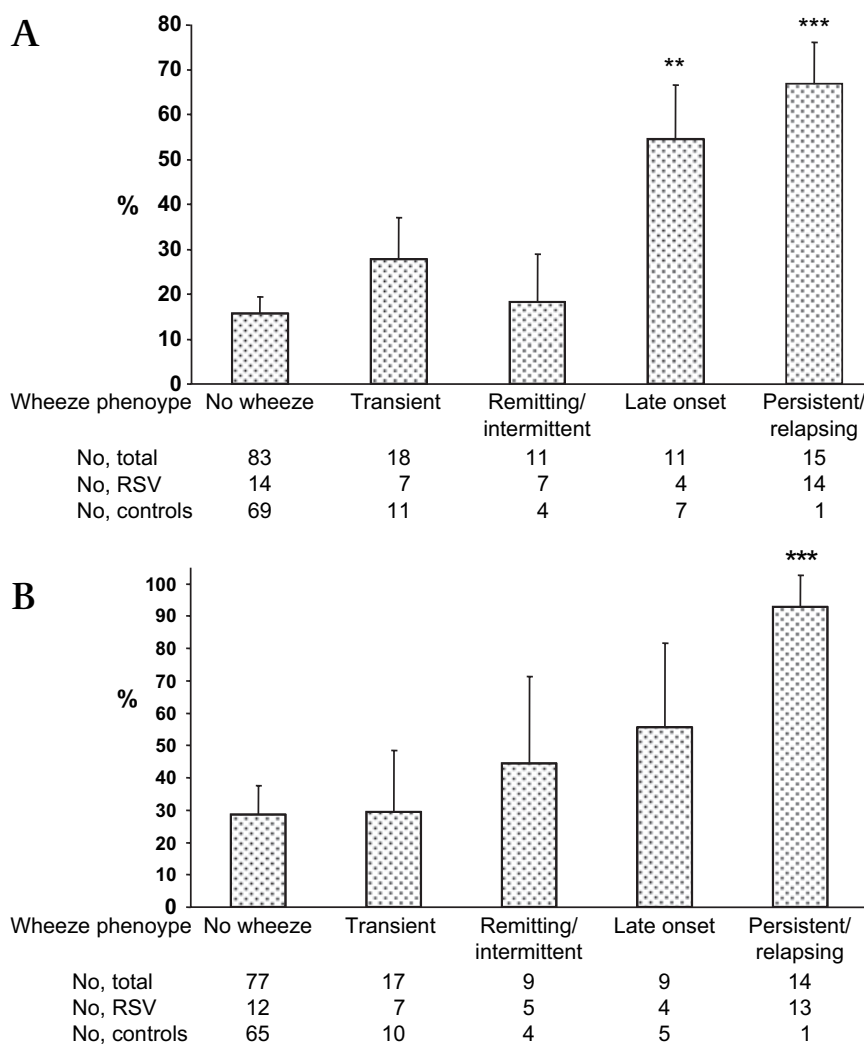

Figure 3 Proportion (\%) of subjects with $(\mathrm{A})$ allergic rhinoconjunctivitis in different wheezing phenotypes (overall $\chi^{2} p<0.001$, ${ }^{* *} p<0.01$, ${ }^{* * *} \mathrm{p}<0.001$ vs the no-wheeze phenotype) and (B) positive Phadiatop test in different wheezing phenotypes (overall $\chi^{2} p<0.001$, ${ }^{* * *} p<0.001$ vs the no-wheeze phenotype). Error bars denote $95 \% \mathrm{CI}$. RSV, respiratory syncytial virus. 
Table 6 Airway function and inflammatory markers in the different wheeze pattern groups for the entire cohort at age 18 years

\begin{tabular}{|c|c|c|c|c|c|}
\hline Variable & Never wheeze & Transient wheeze & Remitting/intermittent wheeze & Persistent/relapsing wheeze & Late onset wheeze \\
\hline Number of subjects & 83 & 18 & 11 & 15 & 11 \\
\hline \multicolumn{6}{|l|}{ Airway function } \\
\hline $\mathrm{FEV}_{1}$ (z-score) & $0.10(0.93)$ & $0.15(1.63)$ & $-0.16(0.97)$ & $-0.80(0.79)^{*}$ & $0.07(0.74)$ \\
\hline $\mathrm{FEF}_{25-75}$ (z-score) & $-0.08(0.83)$ & $-0.16(0.89)$ & $-0.45(0.85)$ & $-0.93(0.73)^{* *}$ & $-0.24(0.85)$ \\
\hline $\mathrm{LCl}$ & $6.54(0.38)$ & $6.52(0.55)$ & $6.46(0.35)$ & $6.81(0.42)^{*}$ & $6.97(0.67)^{* *}$ \\
\hline FeNO (ppb) & $13(6-74)$ & $12(6-34)$ & $21(5-34)$ & $25(7-84)^{*}$ & $18(7-69)$ \\
\hline \multicolumn{6}{|l|}{ Reversibility } \\
\hline Rise in $\mathrm{FEV}_{1}(\%)$ & $3.0(0.0-10.6)$ & $4.8(0.0-10.2)$ & $6.1(0.6-10.2)$ & $6.7(0.0-10.2)^{*}$ & $5.4(0.0-7.8)$ \\
\hline \multicolumn{6}{|l|}{ Inflammatory markers } \\
\hline Blood eosinophils $\left(\times 10^{9} / \mathrm{I}\right)$ & $0.13(0.01-0.79)$ & $0.18(0.04-0.72)$ & $0.16(0.09-0.20)$ & $0.30(0.06-0.57)^{* *}$ & $0.19(0.04-0.47)$ \\
\hline
\end{tabular}

why we do not feel that selection bias significantly affected our results. First, the RSV cohort had the same ARC and asthma parental/sibling heredity prevalence as the controls, and the RSV and control cohorts had a similar prevalence of AD. Second, the control group had a similar prevalence of current asthma/RW, ARC and positive SPT at age 13 to a recent population-based sample of $12-13$-year-old Swedish children living in an overlapping geographical area $\left(8.5 \%, 17 \%\right.$ and $22.5 \%$, respectively). ${ }^{30} 31$ Our study was intended to be a descriptive study and, as such, was not powered to detect differences in hereditary rates of allergic disease/asthma between asthmatic and non-asthmatic index cases. Fifty index cases in each group would have been required to achieve adequate power. Nevertheless, there are indications that early severe RSV bronchiolitis is associated with a greater increased risk for subsequent asthma, clinical allergy and allergic sensitisation when combined with a history of parental asthma (figure $1 \mathrm{~A}-\mathrm{D}$ ).

The $\mathrm{FEV}_{1} / \mathrm{FVC}$ ratio and the $\mathrm{FEF}_{25-75}$, the most reliable and the most sensitive spirometric indices of airway obstruction, respectively, were reduced both at baseline and post-bronchodilator in our RSV cohort. Airway obstruction was most pronounced in subjects with a persistent/relapsing wheezing pattern at age 18, and this pattern could be traced back to ages 7 and 13 years. Interestingly, spirometric indices were also reduced in subjects with RSV without current asthma. These changes may reflect premorbid airway function ${ }^{32}$ or the presence of airway remodelling due to a severe RSV infection occurringduring a critical period of lung development, with or without subsequent interaction with early allergic sensitisation. ${ }^{2} 33$ Young age at first wheezing episode is an important risk factor for subsequent persistent asthma in sensitised cohorts. ${ }^{34}$

In the present study LCI was increased in both RSV and control subjects with current asthma, while spirometry results were impaired only in the subjects with RSV. Increased LCI can result from non-uniformity of ventilation distribution among lung regions sharing branch points in the conducting airway zone or even more peripherally in the vicinity of the terminal bronchioles. ${ }^{3536}$ These results suggest that residual small airway dysfunction is not a result of RSV infection per se but is related to a subsequent diagnosis of asthma. Peripheral airway obstruction and the patchy distribution of airway disease are both recognised characteristics of asthma. ${ }^{9} 37$ Our findings are consistent with other studies documenting raised LCI, despite normal spirometry, in subjects with current mild asthma aged $5-15$ years, ${ }^{38}$ and normal small airway function in an RSV cohort without asthma followed up at age 10 using singlebreath nitrogen washout. ${ }^{5}$ The marked alveolarisation and completion of distal airway formation occurring during the first three years of life may compensate for the more peripheral airway sequelae of early severe RSV infection, while more proximal changes persist. Increased AHR was present in the subjects with asthma, although not to the magnitude previously reported in other studies, in part due to the decision not to withhold ICS prior to testing. A fall in $\mathrm{FEV}_{1}$ of $10 \%$ or more (mean 22.1\%) was seen in 8 of 41 subjects with RSV and in 5 of 86 tested controls (mean 20.1\%; $\mathrm{p}=0.038 ; \chi^{2}$ with Yates' correction). Despite this, a significant correlation of LCI but not spirometry with AHR was ween in our RSV cohort and confirms the important findings of Downie et al. ${ }^{8}$ LCI but not spirometry was also significantly correlated with ongoing airway inflammation (FeNO).

In summary, this study shows that severe primary RSV bronchiolitis in the first year of life is frequently followed by allergic asthma persisting into early adulthood. Subjects with RSV with and without current asthma/RW have reduced airway function as measured by spirometry. Ventilation inhomogeneity, a measure of small airway function, is normal in subjects with RSV without current asthma but is linked to current asthma, AHR and ongoing airway inflammation. Our findings suggest that early severe RSV bronchiolitis has lifelong consequences of allergic asthma and airway remodelling.

Acknowledgements The authors thank Mrs Gunilla Holmgren Wallmyr for skilful assistance at all follow-up visits in this study and Mr Salmir Nasic for statistical advice.

Funding The study was supported by grants from the Regional Health Care Authority of West Sweden, from Borås Hospital, the Fokus Foundation and SeBe's Fund.

\section{Competing interests None.}

Ethics approval This study was conducted with the approval of the Human Research Committee of the Medical Faculty at the University of Gothenburg. All parents and subjects gave consent to the study.

Provenance and peer review Not commissioned; externally peer reviewed.

\section{REFERENCES}

1. Smyth RL, Openshaw PJ. Bronchiolitis. Lancet 2006;368:312-22.

2. Gelfand EW. Pediatric asthma: a different disease. Proc Am Thorac Soc 2009;6:278-82. 
3. Walton RP, Johnston SL. Role of respiratory viral infections in the development of atopic conditions. Curr Opin Allergy Clin Immunol 2008;8:150-3.

4. Stein RT, Sherrill D, Morgan WJ, et al. Respiratory syncytial virus in early life and risk of wheeze and allergy by age 13 years. Lancet 1999:354:541-5.

5. Pullan CR, Hey EN. Wheezing, asthma, and pulmonary dysfunction 10 years after infection with respiratory syncytial virus in infancy. BMJ (Clin Res Ed) 1982;284:1665-9.

6. Korppi M, Piippo-Savolainen E, Korhonen K, et al. Respiratory morbidity 20 years after rsv infection in infancy. Pediatr Pulmonol 2004;38:155-60.

7. Johnson JE, Gonzales RA, Olson SJ, et al. The histopathology of fatal untreated human respiratory syncytial virus infection. Mod Pathol 2007;20:108-19.

8. Downie SR, Salome CM, Verbanck $S$, et al. Ventilation heterogeneity is a major determinant of airway hyperresponsiveness in asthma, independent of airway inflammation. Thorax 2007;62:684-9.

9. Venegas JG, Winkler T, Musch G, et al. Self-organized patchiness in asthma as a prelude to catastrophic shifts. Nature 2005;434:777-82.

10. Juniper EF, Frith PA, Hargreave FE. Airway responsiveness to histamine and methacholine: relationship to minimum treatment to control symptoms of asthma. Thorax 1981;36:575-9.

11. Sigurs N, Bjarnason R, Sigurbergsson F, et al. Asthma and immunoglobulin $E$ antibodies after respiratory syncytial virus bronchiolitis: a prospective cohort study with matched controls. Pediatrics 1995;95:500-5.

12. Sigurs N, Bjarnason R, Sigurbergsson F, et al. Respiratory syncytial virus bronchiolitis in infancy is an important risk factor for asthma and allergy at age 7 . Am J Respir Crit Care Med 2000;161:1501-7.

13. Sigurs N, Gustafsson PM, Bjarnason R, et al. Severe respiratory syncytial virus bronchiolitis in infancy and asthma and allergy at age 13. Am J Respir Crit Care Med 2005;171:137-41

14. Swierkosz EM, Flanders R, Melvin L, et al. Evaluation of the Abbott Testpack RSV enzyme immunoassay for detection of respiratory syncytial virus in nasopharyngeal swab specimens. J Clin Microbiol 1989;27:1151-4.

15. Court SD. The definition of acute respiratory illnesses in children. Postgrad Med J 1973;49:771-6.

16. Ruuskanen 0, Ogra PL. Respiratory syncytial virus. Curr Prob/ Pediatr 1993;23:50-79.

17. American Thoracic Society. Standardization of spirometry, 1994 update. Am J Respir Crit Care Med 1995;152:1107-36.

18. Stanojevic $\mathbf{S}$, Wade A, Stocks J, et al. Reference ranges for spirometry across all ages: a new approach. Am J Respir Crit Care Med 2008;177:253-60.

19. Cole TJ, Stanojevic S, Stocks J, et al. Age- and size-related reference ranges: a case study of spirometry through childhood and adulthood. Stat Med 2009;28:880-98.

20. Gustafsson PM, Aurora P, Lindblad A. Evaluation of ventilation maldistribution as an early indicator of lung disease in children with cystic fibrosis. Eur Respir $J$ 2003;22:972-9

21. Schauer U, Hoffjan S, Bittscheidt J, et al. RSV bronchiolitis and risk of wheeze and allergic sensitisation in the first year of life. Eur Respir J 2002;20:1277-83.
22. Castro $\mathbf{M}$, Schweiger $\mathrm{T}$, Yin-Declue $\mathrm{H}$, et al. Cytokine response after severe respiratory syncytial virus bronchiolitis in early life. J Allergy Clin Immunol 2008; 122:726-33.

23. Thomsen SF, van der Sluis S, Stensballe LG, et al. Exploring the association between severe respiratory syncytial virus infection and asthma: a registry-based twin study. Am J Respir Crit Care Med 2009;179:1091-7.

24. Wu P, Dupont WD, Griffin MR, et al. Evidence of a causal role of winter virus infection during infancy in early childhood asthma. Am J Respir Crit Care Med 2008;178:1123-9.

25. Kuehni CE, Spycher BD, Silverman M. Causal links between RSV infection and asthma: no clear answers to an old question. Am J Respir Crit Care Med 2009:179:1079-80.

26. Kusel MM, de Klerk NH, Kebadze T, et al. Early-life respiratory viral infections, atopic sensitization, and risk of subsequent development of persistent asthma. J Allergy Clin Immunol 2007;119:1105-10.

27. Forton JT, Rowlands K, Rockett $\mathrm{K}$, et al. Genetic association study for RSV bronchiolitis in infancy at the $5 \mathrm{q} 31$ cytokine cluster. Thorax 2009:64:345-52.

28. Thomsen SF, Stensballe LG, Skytthe A, et al. Increased concordance of severe respiratory syncytial virus infection in identical twins. Pediatrics 2008:121:493-6.

29. Mallia $\mathbf{P}$, Johnston SL. Respiratory viruses: do they protect from or induce asthma? Allergy 2002;57:1118-29.

30. Hesselmar B, Aberg B, Eriksson B, et al. Asthma in children: prevalence, treatment, and sensitization. Pediatr Allergy Immunol 2000;11:74-9.

31. Hesselmar B, Aberg B, Eriksson B, et al. Allergic rhinoconjunctivitis, eczema, and sensitization in two areas with differing climates. Pediatr Allergy Immunol 2001; 12:208-15

32. Stern DA, Morgan WJ, Wright AL, et al. Poor airway function in early infancy and lung function by age 22 years: a non-selective longitudinal cohort study. Lancet 2007;370:758-64.

33. Illi S, von Mutius $\mathrm{E}$, Lau $\mathrm{S}$, et al. Perennial allergen sensitisation early in life and chronic asthma in children: a birth cohort study. Lancet 2006;368:763-70.

34. Matricardi PM, Illi S, Gruber C, et al. Wheezing in childhood: incidence, longitudinal patterns and factors predicting persistence. Eur Respir J 2008;32:585-92.

35. Crawford AB, Makowska M, Paiva M, et al. Convection- and diffusiondependent ventilation maldistribution in normal subjects. J Appl Physiol 1985:59:838-46.

36. Paiva M, Verbanck S, van Muylem A. Diffusion-dependent contribution to the slope of the alveolar plateau. Respir Physiol 1988;72:257-70.

37. King GG, Eberl S, Salome CM, et al. Differences in airway closure between normal and asthmatic subjects measured with single-photon emission computed tomography and technegas. Am J Respir Crit Care Med 1998;158:1900-6.

38. Macleod KA, Horsley AR, Bell NJ, et al. Ventilation heterogeneity in children with well controlled asthma with normal spirometry indicates residual airways disease. Thorax 2009:64:33-7. 